\title{
Color Barrier Free Displays in Disaster Situations
}

\author{
Kensei Tsuchida, Chieko Kato, Toshiaki Nagshima \\ Faculty of Information Sciences and Arts, Toyo University, Kawagoe, Japan \\ Email: kensei@toyo.jp, kato-c@toyo.jp, to4ak.ngsm1106@kmh.biglobe.ne.jp
}

Received 16 January 2016; accepted 16 February 2016; published 23 February 2016

\begin{abstract}
Concerning color barrier free, there have been many research studies and an increasing interest in it. For example, to expand activities for the promotion of colorblind-friendly designs not only among scientists but also among industrial products and public facilities, the non-profit organization CUDO (Color Universal Design Organization) was established in 2004. However, there has been very little research of this type focusing on disaster situations. From their experiences volunteering in reconstruction support for victims of the 2011 tsunami, the authors came to recognize the importance for both non-colorblind and colorblind people to notice and distinguish emergency escape notifications, cautions and passage maps quickly and accurately. Therefore this research is aimed at the realization of color barrier free displays in disaster situations. Initially, requirements for color barrier free displays in disaster situations were analyzed. Four desirable criteria were derived as a result: "eye-catching", "instantly-readable", "unmistakable" and "stable". In order to evaluate given signs from the viewpoint of the above criteria, evaluation experiment systems for displays in disaster situations were developed. With the evaluation experiment systems it would be possible to design effective signs and maps as color barrier free displays in disaster situations.
\end{abstract}

\section{Keywords}

Color Barrier Free, Design, Displays, Disaster Situations, Evaluation Systems

\section{Introduction}

Color plays important roles in our daily life. People decide what is safe or not, depending on the color. People can gather information by color and enjoy looking at colorful pictures. However more people than we realize are affected by some form of colorblindness. To help people who are colorblind, the concept of CUD (Color Universal Design) was established [1] [2]. CUD uses a combination of different colors which can be easily recognized by all color vision types. The purpose of the CUDO (Color Universal Design Organization) [3] is to improve the design of products, signs and publications, so that people with different kinds of color vision can easily interpret information.

To the present, there have been many research studies and increasing interest in it. For example, to expand activities for the promotion of colorblind-friendly designs not only among scientists but also among industrial 
products and public facilities [4]. However, there has been very little research of this type focusing on disaster situations. From their experiences volunteering in reconstruction support for victims of the 2011 tsunami, the authors came to recognize the importance for both non-colorblind and colorblind people to notice and distinguish emergency escape notifications, cautions and passage maps quickly and accurately.

Therefore the authors aimed at the realization of color barrier free displays in disaster situations. As the first step of the realization of it, requirements for color barrier free displays in disaster situations were analyzed and evaluation experiment systems for displays in disaster situations were developed.

\section{Colorblind and CUDO}

It is common among men to suffer from color blindness. $8 \%$ of Caucasians, $4 \%$ of people of African descent, and $5 \%$ of Asian males are colorblind. It is important for people to be able to identify different colors. Among people who are colorblind, D type (deuteranopia) and $\mathrm{P}$ type (protanopia) are the most common [2]. These people have difficulty distinguishing between reds and greens. P and D type, along with C type (common type), are the three major types of color vision. C type people have normal color vision. Another type of colorblindness is T type (Tritanope type). These people confuse blue and yellow. Also there are some people who are monochromats, completely colorblind.

CUDO chose colors which can be easily identified by people who are colorblind [3]. Their results have been incorporated into the industry standards for color specification in the paint industry, as well as the printing and design industries, and are gaining popularity in their respective fields. Their color scheme set contains 20 colors in all, divided into 3 groups in the JPMA (Japan Paint Manufacturers Association) version, with two alternate colors added for a total of 22. The first of these is the high-saturation accent color group used for letters and signs, and includes 9 colors: red, orange, yellow, green, blue, sky blue, purple, pink, and brown. These colors are to be used when looking at something on a smaller scale. The second group of highly bright colors, used for looking at things on a larger scale, like maps and street guides, includes 7 colors: bright pink, cream, bright green, bright sky blue, beige, bright purple and bright yellow-green. The third group includes the 4 chromatic colors of white, bright grey, grey, and black. The JPMA version adds yellow and green as alternate colors. CUDO chose 7 colors (yellow, red, green, sky blue, purple, pink and blue) that are commonly used by people with normal color vision, and then chose 7 colors from the 9 accent color group and changed them into colors for people who are colorblind.

\section{Requirements for Color Barrier Free Displays in Disaster Situations}

From their experiences volunterring in reconstruction support for victims of the 2011 tsunami, the authors came to recognize the importance for both non-colorblind and colorblind people to notice and distinguish emergency escape notifications, cautions and passage maps quickly and accurately. Requirements for color barrier free displays in disaster situations were analyzed and considered. Four desirable criteria were derived as a result: "eye-catching", "instantly-readable", "unmistakable" and "stable".

- Eye-Catching

Eye-catching implies that people can notice a specified sign at once among various objects or displays.

- Instantly-Readable

Instantly-readable implies that people can comprehend the contents of signs without taking much time.

- Unmistakable

Unmistakable implies that signs do not give misleading information to people. For instance, it is desirable to avoid ambiguous signs.

- Stable

Stable implies that people can read signs easily and stably under varied circumstances.

\section{Evaluation Experiment Systems for Displays}

In order to evaluate given signs from the viewpoint of the above criteria, evaluation experiment systems for displays in disaster situations were developed. The development language was HSP (Hot Soup Processor programming language) and the development environment was Windows 8.

The evaluation experiment systems have the following features (1)-(4). 
(1) With the systems, examinees can take a color barrier free test like playing some kind of an easy game.

(2) To take account of order effect, the systems give random problems to examinees.

(3) The systems can measure response time of examinee's answering and calculate the accuracy rate of it.

(4) Experimenters can easily customize the systems. That is, it is easy to change backgrounds and display objects only by replacing relevant picture image files.

With the systems, it is possible to evaluate displays from the view point of eye-catching, instantly-readable, unmistakable and "stable" by the feature (3) and changing color lighting and brightness.

Figures 1-6 are screen shots of windows executing the systems. Figure 1 shows a window after 3 seconds from starting. Figure 2 shows a window in which one example notice board and six alternatives are presented. An examinee has to click the same notice board as the example from the six alternatives quickly and accurately. In Figure 2 a problem without background is presented. In Figure $\mathbf{3}$ and Figure $\mathbf{4}$ the backgrounds are an underground passage and a park respectively.

In Figure $\mathbf{5}$ a problem of only colored characters is presented. In Figure $\mathbf{6}$ an evaluation result is presented. The result reports correct/incorrect and response time for each problem.

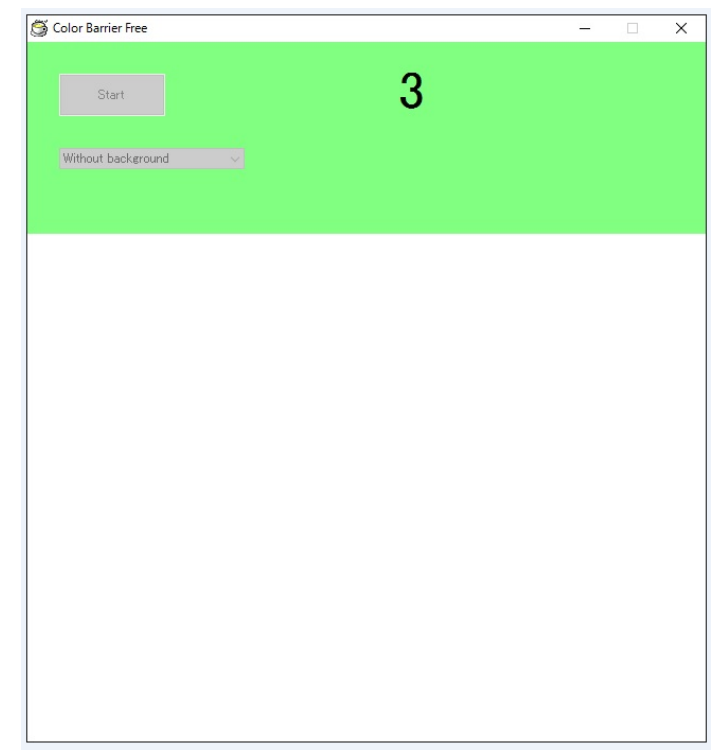

Figure 1. A window after 3 seconds from starting.

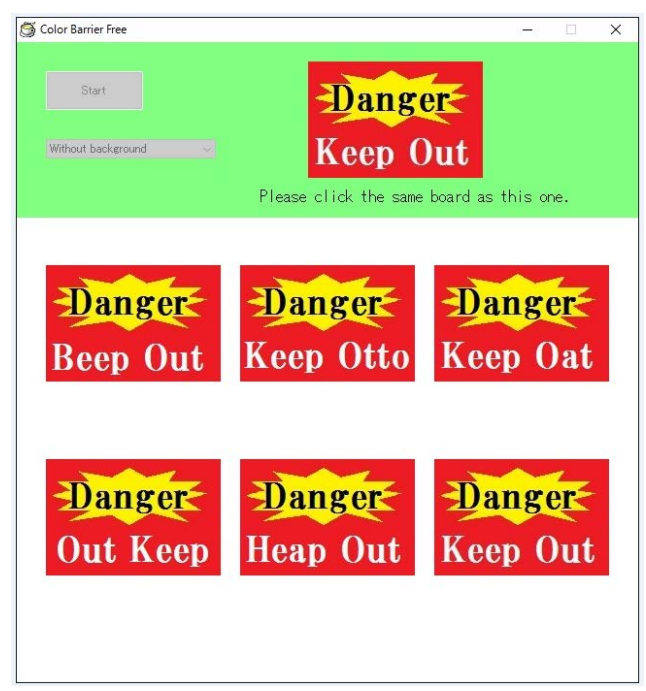

Figure 2. A problem without background. 


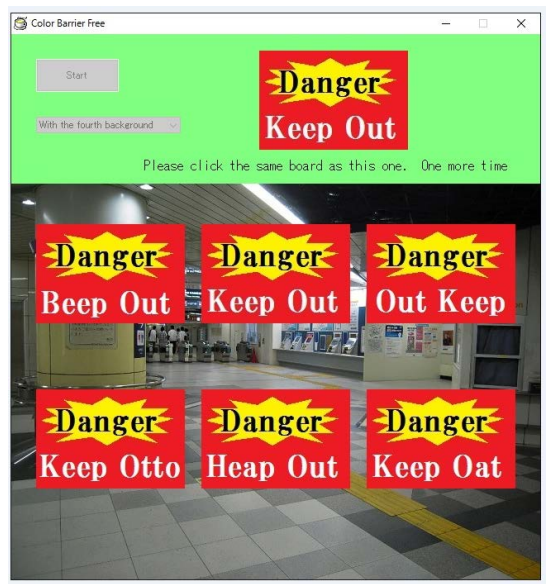

Figure 3. With a background of an underground passage.

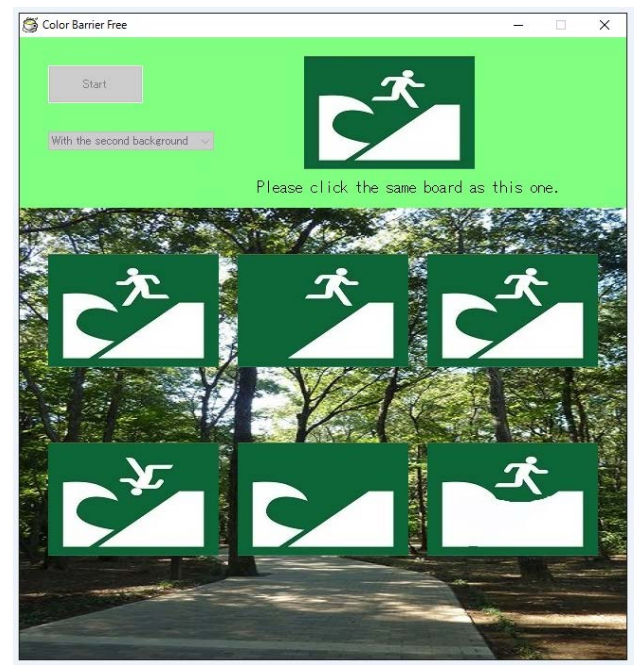

Figure 4. With a background of a park.

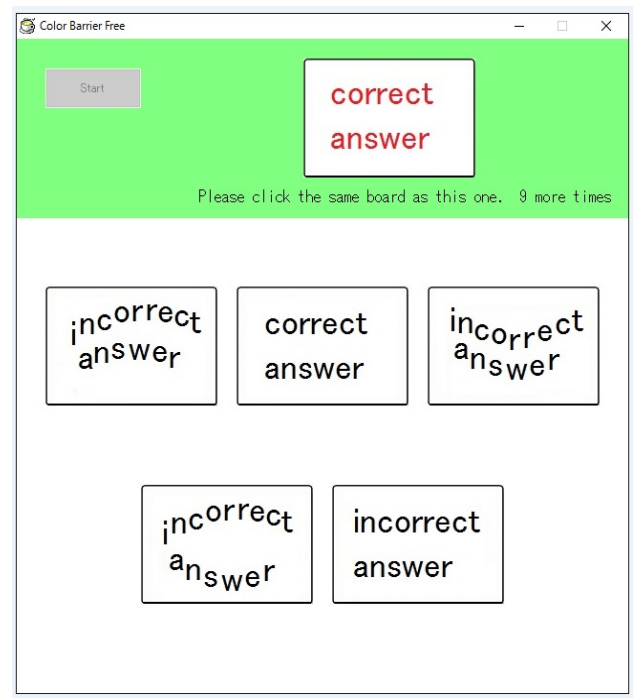

Figure 5. A problem of only colored characters. 


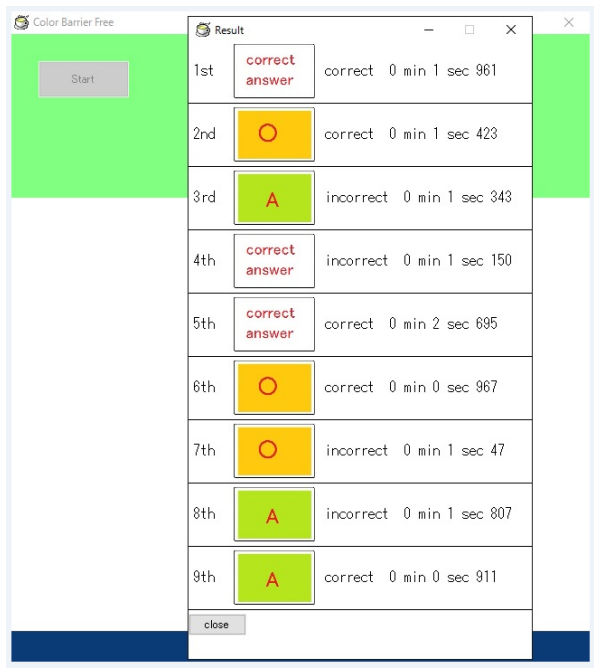

Figure 6. An evaluation result.

\section{Conclusions}

In this study, requirements for color barrier free displays in disaster situations were analyzed and considered. Four desirable criteria were derived as a result: "eye-catching", "instantly-readable", "unmistakable" and "stable". In order to evaluate given signs from the viewpoint of the above properties, evaluation experiment systems for displays in disaster situations were developed. The systems can measure response time of examinee's answering and calculate the accuracy rate of it. This makes it possible to evaluate given signs from the view point of color barrier free objectively and quantitatively.

In future this study needs to make repeated experiments of evaluation of color barrier free displays with developed systems.

\section{References}

[1] Ichihara, Y.G., Okabe, M., Iga, K., Tanaka, Y., Musha, K. and Ito, K. Color Universal Design-The Selection of Four Easily Distinguishable Colors for all Color Vision Types. Preprint for the 2008 The Society for Imaging Science and Technology Meeting at San Jose.

[2] http://jfly.iam.u-tokyo.ac.jp/lab/colorresearch.html

[3] http://www.cudo.jp/ (in Japanese)

[4] Eizo Nanao Corporation, Color Universal Design Handbook. http://www.iar.unicamp.br/lab/luz/ld/Cor/Color\%20Universal\%20Design\%20handbook.pdf 